ESAIM : Proceedings, Vol. 7, 1999, 1-11

Third International Workshop on Vortex

Flows and Related Numerical Methods

http://www.emath.fr/proc/Vol.7/

\title{
Vorticity evolution on a separated wavy wall flow
}

\author{
C. Airiau, A. Giovannini \\ Institut de Mécanique des Fluides de Toulouse, UFR MIG, Université Paul Sabatier, \\ 118 Rte de Narbonne, F31062 Toulouse Cedex, FRANCE. \\ E-mail : Christophe.Airiau@imft.fr, giova@imft.fr
}

\begin{abstract}
This paper is concerned by the study of 2D incompressible and unstready flow over a wavy wall. The numerical procedure is based on the full Random Vortex Method algorithm. The use of a conformal mapping make it gridless. Separation and reattacheemnt mechanisms are tackled through the Lighthill mechanism relating the streamwise pressure gradient with the injected flux of vorticity at the wall. Different post-processing for the pressure and the wall shear stress allow the caracterization of unsteadiness sources in the shear layer and the recirculation. Finally drag reduction by considering the competition between the pressure drag and the viscous drag is adressed.
\end{abstract}

\section{Introduction}

This paper is devoted to the study of an external two-dimensional incompressible wavy wall flow using the Random Vortex Method. We have focused our analysis on the vorticity near the wall induced by the streamwise pressure gradient.

Two parameters characterize this complex flow : a geometric factor $\eta=\frac{2 a}{\lambda}$ where $2 a$ is the crest to crest wavy wall amplitude and $\lambda$ the spatial periodicity (wavelength), and the Reynolds number $R e=\frac{U_{\infty} \lambda}{\nu}$ based on the reference velocity far from the wall. In order to have a transitional regime and separated flow, these parameters are fixed to $\eta=0.05$ and $R e=70000$.

A wavy wall appears to be an interesting geometry for the investigation of drag reduction since on one portion of the surface the skin friction coefficient changes sign. On the other hand, the redistribution of pressure due to separation and reattachment events increases the pressure drag. Both of these components of the drag, as shown later in the paper, are directly proportional to the vorticity at the wall and to its normal flux. Hence, the vorticity plays a major role on the drag phenomenon. Another field of industrial interest may be found in the enhancement of heat transfer. But this was not done in this present work.

Most of previous numerical, theoretical or experimental studies implying wavy wall concern channels flows.

Zilker and Hanratty (1979) gave a diagram $(R e, \eta)$ describing the existence of separated flow and measured the evolution of the wall pressure distribution $C_{p}$.

The pressure plateau in the case of separation is confirmed by Buckles et al (1984), and they remark that the region of reversed flow varies with time dramatically in size compared to the case 
of laminar separated flows. The intermittency of the reattachment point in forward and reverse direction is important. It is shown that the intermittency of the reversed flow is 80 percent of the time $(x / \lambda=0.4)$ but only 10 percent at $(x / \lambda=0.4)$.

Chauve and Schiestel (1985) in the range $30000<R e<115000$ and for $\eta=0.1$ solved the Navier-Stokes equations with turbulence modeling and found agreement with their own experiments concerning the small recirculation zone.

More recently, Blancher et al (1994), Patel et al (1991) found recirculation zones filling the whole cavities for different flow regimes.

External flows have been investigated by Caponi et al (1982) for $R e=40000$ and $\eta=0.1$, using a stream-function - vorticity formulation; by Tsangaris et al (1986) using perturbation methods for a very low Reynolds number; and by Saidi et al (1987) for $R e=16000$ and $\eta=0.1$ In both of these studies recirculation zones of different sizes appear and increase with the Reynolds number.

We can conclude from this brief review that a large bibliography exists for internal flows, with different channel geometries, but no major and complete studies were performed for external unsteady flow with an amplitude large compared to the boundary layer thickness.

The first section of this paper describes briefly the numerical method (RVM) and gives the numerical parameters ensuring convergence of the calculations.

The second section gives details on the post-processing calculation of the strain tensor component (pressure field and shear stress at the wall).

In the third section, the averaged and instantaneous pressure, stream-function and velocity fields are analyzed. The Lighthill mechanisms is shown, emphazising the relationship between the streamwise pressure gradient and the wall vorticity.

\section{Numerical method}

The two-dimensional incompressible Navier-Stokes are solved with the vorticity-velocity formulation. The reference length is the spatial wall periodicity $\lambda$, the reference velocity is the stream-wise velocity $U_{\infty}$. The curl of the velocity has only one component $\omega$, solution of the non-dimensional equation :

$$
\frac{\partial \omega}{\partial t}+(\vec{U} \cdot \nabla) \omega=\frac{1}{R e} \Delta \omega
$$

The RVM algorithm (Chorin,1973, Gagnon et al, 1993) is used. The solution is approximated by calculating the motion of a finite number of two kinds of vortex elements. In the bulk of the flow they are vortex blobs of radius $r_{0}$, regularized by Chorin's kernel, and close to the solid wall they are vortex sheets (Chorin,1978).

The convection step $\frac{d \omega}{d t}=0$ which corresponds to the Euler equation is solved without a grid by providing for the potential flow an analytical solution. A conformal mapping $z=H(Z)=$ $Z+a / \lambda \exp (2 i \pi Z)$ provides a correspondence between the physical plane $(z=x+\imath y)$ and the transformed half-plane $(Z=X+\imath Y, Y \geq 0)$. The hydrodynamic images ensure that at the wall 
there is a slip velocity of the vortical part of the velocity field. The vortex element velocities are then evaluated by Routh law (Clements, 1973) giving for the element $\left(\Gamma_{j}, z_{j}, Z_{j}\right)$ :

$$
w\left(z_{j}\right)=u-\imath v=\frac{1}{H^{\prime}\left(Z_{j}\right)}\left(1-2 \imath \sum_{k \neq j}^{n v}\left(\frac{\gamma_{k}}{Z_{j}-Z_{k}}-\frac{\gamma_{k}}{Z_{j}-\bar{Z}_{k}}\right)+\imath \gamma_{j} \frac{H^{\prime \prime}\left(Z_{j}\right)}{H^{\prime}\left(Z_{j}\right)}\right.
$$

where $\gamma_{j}=\frac{\Gamma_{j}}{4 \pi U_{\infty} \lambda}$ is the dimensionless circulation of the element $j$. Primes denote derivative with respect to $Z$. The vortex blob displacement is obtained by integrating in time the convection velocity.

The no-slip condition is satisfied in local $(\vec{t}, \vec{n})$ coordinates, at some control points $S w_{i}$ of the wavy wall. The generation of the vorticity is made through vortex sheet elements with a constant density of circulation $\gamma=\frac{d \Gamma}{d s}$ and a length $h$. At each time step, the spurious velocity at the wall $U_{\mathrm{W}}$ which is observed at the end of the convection step induces a vorticity flux $\delta \Phi_{w_{i}}$ (see section 4.1) according to the relation :

$$
\delta \Phi_{w_{i}} \Delta t=\int_{S w_{i-\frac{1}{2}}}^{S w_{i+\frac{1}{2}}} \int_{t}^{t+\Delta t} \varphi_{\mathrm{W}} d t d s=U_{\mathrm{W}} h=-\Gamma=-\gamma h
$$

The slip velocity then is annihilated by injection of an adequate number of vortex sheets in the flow.

The diffusion step which corresponds to the equation

$$
\frac{\partial \omega}{\partial t}=\frac{1}{R e} \Delta \omega
$$

is also handled by a lagrangian formulation : each vortex element undergoes a random walk $\left(\eta_{x}, \eta_{y}\right)$ with gaussian distributed random numbers. This mimics the Green function distribution of the heat equation.

The following numerical parameters for the discretisation of time, space and circulation ensure the numerical convergence of the algorithm (see Sethian and Ghoniem, 1988, Mortazavi et al, 1996) :

$$
\Delta t=0.05 \quad \gamma_{j}=0.002 \quad h=\pi r_{0}=0.1
$$

These parameters generate 7500 vortex sheets and 16000 vortex blobs. The convergence was established by the criteria of a quasi constant number of particles after a certain time, and by plotting the mean and steady velocity. Under a certain range, the choice of another value of the numerical parameters have provided the same mean velocity profile and size of mean recirculation zone, but sometimes with less accuracy or more particles (but with a great increasing of the computational time). The establishment of the regime is numerically reached after 300 time steps, corresponding to a non-dimensional time $\tau=15$. The analysis in section 4 is made over 512 consecutive time steps, between $\tau=25.6$ and $\tau=45$. The resolution is performed over 900 time steps without significant evolution of the statistics. 


\section{Post-processing of the vorticity, velocity and pressure fields}

\subsection{Pressure field}

A post-processing procedure is required to calculate onto a grid of moderate size $(60 \times 60)$ the pressure field. The velocity field must be first of all, calculated at each time step, at the grid points. The potential part of the velocity $\vec{U}_{p}$ is given by the conformal transformation and the rotational part $\vec{U}_{\omega}$ is obtained with the Biot-Savart law. It is important to outline that these analytical evaluations are very accurate compared numerical evaluations, and keep the velocity divergence free. The pressure field is the solution of the Poisson equation obtained by taking the divergence of the momentum equation :

$$
\frac{\partial \nabla \cdot \vec{U}}{\partial t}+\operatorname{tr}(\overline{\bar{\nabla}} \vec{U} \otimes \overline{\bar{\nabla}} \vec{U})+\vec{U} \cdot \vec{\nabla}(\nabla \cdot \vec{U})=-\Delta p+\frac{1}{R e} \nabla \cdot(\vec{\nabla}(\nabla \cdot \vec{U}))
$$

Using the divergence free condition, this leads to

$$
\Delta p=-\operatorname{tr}(\overline{\bar{\nabla}} \vec{U} \otimes \overline{\bar{\nabla}} \vec{U})
$$

In two-dimensional flows, the pressure equations becomes :

$$
\Delta p=-2\left[\frac{\partial U_{y}}{\partial x} \frac{\partial U_{x}}{\partial y}+\left(\frac{\partial U_{y}}{\partial y}\right)^{2}\right]=F(x, y, t)
$$

A Dirichlet condition $(p=0)$ is imposed far from the wall $\left(y=y_{\max }\right)$. Neumann conditions are fixed for the three other boundaries (inlet,outlet,wall). These conditions come from the normal projection of the momentum equation :

$$
\vec{\nabla} p \cdot \vec{n}=\vec{G}(x, y, t) \cdot \vec{n}=g(x, y, t)
$$

The right-hand side term is known from the RVM calculation. The physical domain is transformed into a regular computational grid by the mapping :

$$
\xi=x \quad \eta=1-\exp \left[\frac{y_{\mathrm{w}}(x)-y}{y_{0}}\right]
$$

The transformed system of P.D.E. in $(\xi, \eta)$ for the pressure equation and the boundary conditions are discretized with a second order finite difference scheme in space and time, since time derivative are present in the boundary condition $g(x, y, t)$.

A Point Successive Under Relaxation (PSUR) algorithm solves the system of linear equations :

For the inner domain

$$
p_{i j}^{k+1}=p_{i j}^{k}+\omega_{0} \phi\left(p_{h l}^{k}, p_{h l}^{k+1}, F_{i j}\right)
$$

and for the boundaries :

$$
p_{i j}^{k+1}=p_{i j}^{k}+\omega_{1} \psi\left(p_{h l}^{k}, p_{h l}^{k+1}, g_{i j}\right)
$$


$\omega_{0}$ and $\omega_{1}$ are relaxation factors which lay between 0.5 and 0.8 .

Through different tests on basic flows (Blasius boundary layer, unsteady and steady Poiseuille flow, potential wavy wall flow), it appears that the implementation of the relaxation in the boundary condition treatment improves significantly the rate of convergence and the accuracy of the solution at the frontiers of the domain. At the first step $(k=1)$, the initial guess of the pressure field is built by integrating the momentum equation in the $y$ direction, from the upper boundary to the wall.

Convergence of the PSUR algorithm is achieved when $\left|p_{i j}^{k+1}-p_{i j}^{k}\right|<10^{-5}, \quad \forall i, j$

\subsection{Skin friction coefficient at the wall and total drag}

This coefficient is defined in dimensionless variables as : $C f=\frac{\tau_{w}}{\frac{1}{2} \rho U_{\infty}^{2}}$ where $\tau_{w}$ is the tangential viscous stress at the wall. With a flat wall, with dimensionless quantity, it is given by:

$C f=-\left.\frac{2}{R e} \frac{\partial U}{\partial y}\right|_{\text {wall }}=\frac{2}{R e} \omega_{\text {wall }}$. With a wavy wall, the tangential viscous stress is function of the wall slope $y_{\mathrm{W}}^{\prime}(x)=\tan \theta$ and the coefficient becomes:

$$
\left.C f=\frac{2}{R}\left[\cos 2 \theta\left(\frac{\partial U_{x}}{\partial y}+\frac{\partial U_{y}}{\partial x}\right)+2 \sin 2 \theta \frac{\partial U_{y}}{\partial y}\right)\right]_{\text {wall }}
$$

The value of the skin friction coefficient is accurately calculated using a second order finite difference scheme at the wall.

The dimensionless total drag is given by equation :

$$
C_{D}=\frac{F_{x}}{\frac{1}{2} \rho U_{\infty}^{2} \lambda}=\int_{x_{0}}^{x_{1}}\left(y_{w}^{\prime}(x) C p(x)+\frac{2}{R e}\left[\frac{\partial U_{x}}{\partial y}+\frac{\partial U_{y}}{\partial x}+2 y_{w}^{\prime}(x) \frac{\partial U_{y}}{\partial y}\right]_{\text {wall }}\right) d x
$$

\section{Analysis in terms of vorticity and results}

The goal of this section is to focus on the relation between the vorticity and its normal derivative and the wall component of the stress tensor, following the ideas of Lighthill.

\subsection{Vorticity and vorticity flux}

The vorticity $\vec{\Omega}=\vec{\nabla} \wedge \vec{U}$ in three-dimensional constant density flows is present in the momentum equation and naturally in the vorticity transport equation respectively under the terms $\mu \vec{\nabla} \wedge \vec{\Omega}$ and $\nu \Delta \vec{\Omega}$. In analogy with the case of heat flux, Lighthill has defined the vorticity flux tensor $\overline{\bar{\varphi}}_{\omega}=-\nu \overline{\bar{\nabla}} \vec{\Omega}$. The divergence $\nabla \cdot \overline{\bar{\varphi}}_{\omega}$ gives the diffusion term in the vorticity transport equation.

Considering a volume of fluid and its surface with a normal vector $\vec{n}$, the volume and surface integrals correspond to the viscous force $\vec{F}_{v}$ and to the vorticity flux $\vec{\Phi}_{\omega}$ as :

$$
\vec{F}_{v}=-\mu \int_{\mathcal{V}} \vec{\nabla} \wedge \vec{\Omega} d \mathcal{V}=-\mu \int_{\mathcal{S}} \vec{n} \wedge \vec{\Omega} d \mathcal{S}
$$




$$
\vec{\Phi}_{\omega}=\nu \int_{\mathcal{V}} \Delta \vec{\Omega} d \mathcal{V}=-\int_{\mathcal{S}} \overline{\bar{\varphi}}_{\omega} \cdot \vec{n} d \mathcal{S}
$$

Finally, one can simplify these general relations in the case of two-dimensional flows because the vector $\vec{\Omega}$ is reduced to one component. At the solid wall, the viscous tangential stress is

$$
\tau_{\mathrm{W}}=-\mu \omega_{\mathrm{wall}}
$$

and the relationship between the vorticity flux and the streamwise pressure gradient is

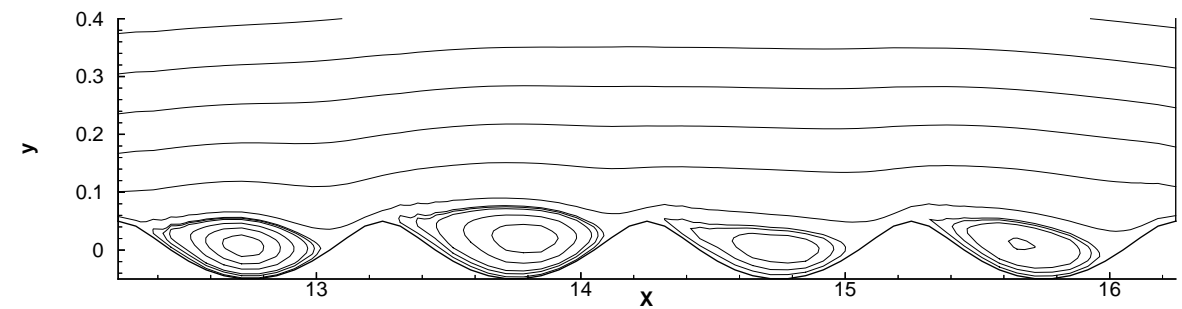

a)

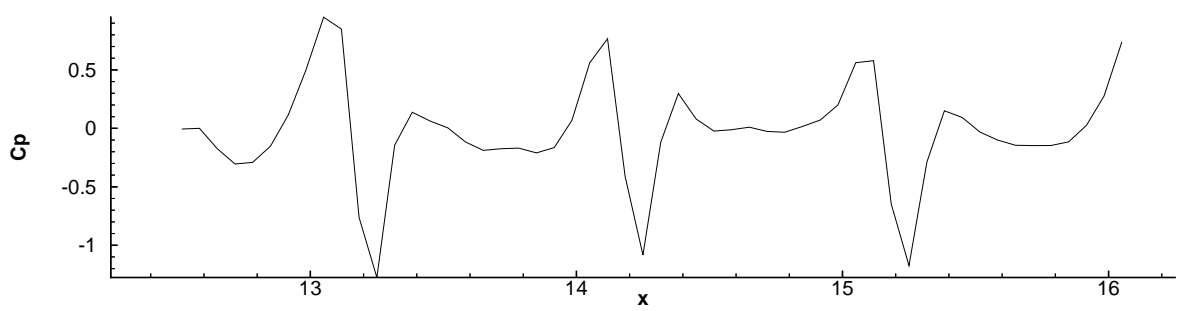

b)

Figure 1: a) : Averaged stream-function; b) : Averaged wall pressure coefficient

$$
\varphi_{\mathrm{W}}=-\left.\nu \frac{\partial \omega}{\partial n}\right|_{\mathrm{wall}}=\left.\frac{1}{\rho} \frac{\partial p}{\partial s}\right|_{\mathrm{wall}}
$$

\subsection{Results}

\subsubsection{Mean flow}

The calculations are performed over a domain of length equal to four wavelengths. Here we present averaged quantities over 512 time steps.

Figure (1a) and (1b) shows the mean stream-function and the mean wall pressure coefficient $C p$. No numerical periodic boundary conditions are imposed but a spatial periodicity seems to exist after the second cavity.

The evolution of the mean pressure (fig. 2) in the total flow shows that the normal to the wall component of the pressure gradient is negligible compared to the streamwise pressure gradient. It means that the streamwise pressure gradient $\frac{\partial p}{\partial s}$ at the wall follows the evolution of the dividing streamline $\left(\psi=\psi_{0}=0\right.$, see figures 3 and $\left.4 \mathrm{a}\right)$ which separates the outer flow from the recirculation zone. The streamwise pressure gradient is equal to the value obtain by a potential flow with a virtual solid wall given by the streamline $\psi_{0}$. According to this fact, the maximum of pressure 


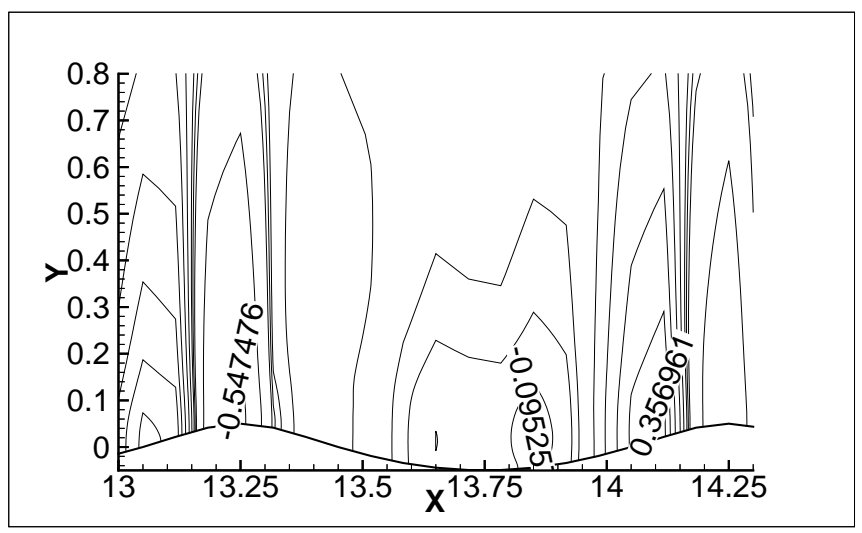

Figure 2: Averaged pressure in the second cavity

occurs at the reattachment point (the vorticity at the wall is equal to 0 ), and the minimum occurs just before the separation point.

Now, let us just consider the motion over the second cavity which appears to be "typical". Figures (4a to $4 \mathrm{~d}$ ) respectively show the stream-function, the wall pressure coefficient, the wall vorticity and the wall vorticity flux.

The Lighthill mechanism explains the birth of the separation and of the reattachment. First, the flow is mainly irrotational and vorticity is equal to zero everywhere, except near the wall where negative vorticity is injected from the wall. But due to the sign of the streamwise pressure gradient, after the crest, positive vorticity is injected. Separation occurs when the injection of positive vorticity is equal to the negative vorticity of the boundary layer. Vorticity is zero at the separation point (point $\mathrm{S}$ ). Then little vorticity is injected in the recirculation zone, and vorticity stays approximately constant, until the potential streamwise pressure gradient decreases, inducing the injection of negative vorticity. Vorticity decreases, reaches zero at the reattachment point (point R). Then the pressure gradient is negative, and negative vorticity is injected in a new boundary layer.

Friction drag decreases due to the negative wall viscous stress in the recirculation zone, but pressure drag increases dramatically due to the separation streamline $\psi_{0}$. Finally, the total drag is greater than in the case of a Blasius flow.

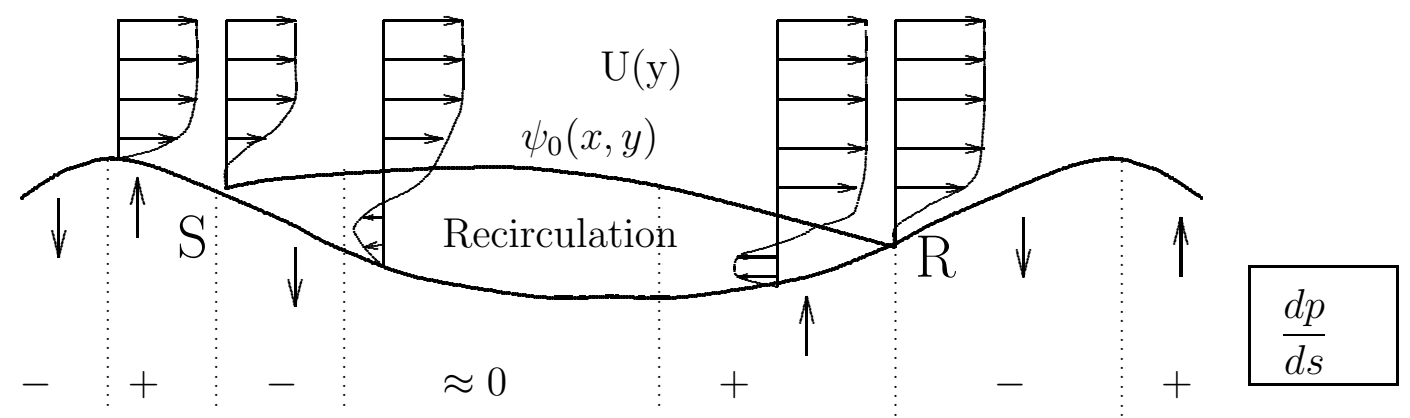

Figure 3: vorticity flux and pressure gradient at the wall 


\subsubsection{Unsteady flow}

Instead of studying the unsteady total velocity, pressure and vorticity, we have chosen to investigate the evolution of fluctuation values defined by

$$
q=Q(x, y, t)-<Q(x, y)>_{T}=Q(x, y, t)-\frac{1}{T} \int_{t_{0}}^{t_{0}+T} Q(t) d t
$$

All the fluctuations were investigated. We will present only the results for the vorticity field.

Since wall vorticity is equal to zero at the separation and reattachment point, it is very sensitive to small perturbations coming from the outer flow and to the streamwise pressure gradient. This means that the location and the size of the recirculation zone are very unstable to pressure and velocity perturbations. The result is that alternatively, due to the variation of pressure gradient, injection of negative and positive vorticity occurs between the crest and the separation point. These small "bursts" induce unsteadiness in the flow. These perturbations of vorticity follow three paths. A first part increases or decreases vorticity in the cavity, and so contributes to the unsteadiness of the reattachment point. A second part, the main part, is ejected and convected in the outer flow. This second part, is divided into two parts when it goes above the next cavity. One contributes to the instability of the separation point, and the rest disappears by viscous dissipation, or is convected in the free stream outside the separation zones.

We may notice that experimentally such a behavior is not observed. In turbulent channel wavy wall flow, the unsteadiness of the recirculation zone is seen, but the event is too rapid and the recirculation zone is too small to verify that point. Actually, one may think that our results show the large transient instability that triggers the transition of the flow to turbulence. It is certain that recirculation zone creates at least strong inflexional instability.

\section{Conclusion}

Full Lagrangian Random Vortex Method using conformal mapping is an effective tool to investigate unsteady separation of wavy wall flow with a high level of accuracy and a large time steps.

Separation mechanism may be explained and understood through the Lighthill mechanism i.e. the relation between the streamwise pressure gradient and the wall vorticity flux.

The numerical results obtained give the physical evolution of the flow. In particular, the strong unsteadiness of the recirculation zone will induce transition to turbulence. It is clear that the wavy wall does not reduce the total drag, because of the streamwise pressure gradient which increases drastically the pressure drag.

\section{References}

1 S. Blancher, R. Creff, J. Batina, P. André : Hydrodynamic stability in periodic geometry. Finite elements in analysis and design. $16,261-270$ (1994)

2 J. Buckles ,T. J. Hanratty, R. J. Adrian : Turbulent flow over large-amplitude wavy surfaces. J. Fluid Mech. 140,27-44 (1984)

ESAIM: Proc., Vol. 7, 1999, 8 -11 
3 E.A. Caponi, B. Fornberg, D.D. Knight, J.W. Mclean, P.G. Saffman, H.C. Yuen : Calculations of laminar viscous flow over a moving wavy surface. J. Fluid Mech.124, 347-362 (1982)

4 M.P. Chauve, R. Schiestel : Influence of weak wall undulations on the structure of turbulent pipe flow : an experimental and numerical study. J. Fluid Mech.160, 47-75 (1985)

5 A.J. Chorin : Numerical study of slightly viscous flow. J. Fluid Mech. 57 (1973)

6 A.J. Chorin : Vortex sheet approximation of boundary layers. J. Comput. Phys. 27,423 (1978)

7 R.R. Clements : An inviscid model of two-dimensional vortex shedding. J. Fluid Mech. 57, part 2, 321-336 (1973)

8 Y. Gagnon, A. Giovannini, P. HÉBrard : Numerical simulation and physical analysis of high Reynolds number recirculating flows behind sudden expansions. Phys. Fluids, A 5 , (10), 2377-2389 (1993).

9 A. F. Ghoniem , Y. Gagnon : Vortex simulation of laminar recirculating flow . J. Comp. Phys. 68 , ( 2 ), 346-377 ( 1987 )

10 I. Mortazavi, P. Micheau, A. Giovannini : Numerical convergence of the Random Vortex Method for complex flows ESAIM: Proceedings, Vol.1. Vortex Flows and Related Numerical Methods II, http://www.emath.fr/proc/Vol.1/ (1996)

11 C. Saidi, F. Legay-Desesquelles, B. Prunet-Foch : Laminar flow past a sinusoidal cavity. Int. J. Heat Mass Transfer, 30 , (4), 649-661 (1987)

12 J. A. Sethian , A. F. Ghoniem : Validation study of vortex method . J. Comp. Phys. 74, $283-317$ (1988)

13 S. Tsangaris, D. Potamitis : On laminar small Reynolds Number flow over wavy wall. Acta Mechanica $61,109-115(1986)$

14 D. P. Zilker, T. J. HAnRAtTy : Influence of the amplitude of a solid wavy wall on a turbulent flow. Part 2. Separated flows. J. Fluid Mech. 90, 257-271 (1979) 


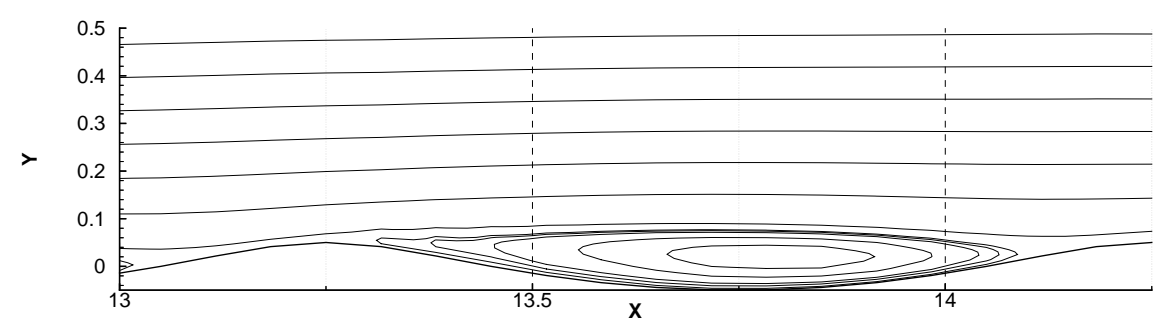

a)

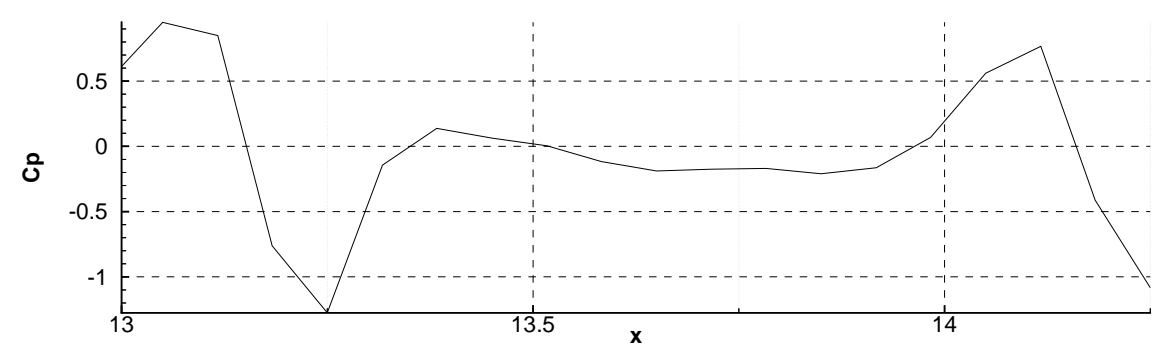

b)

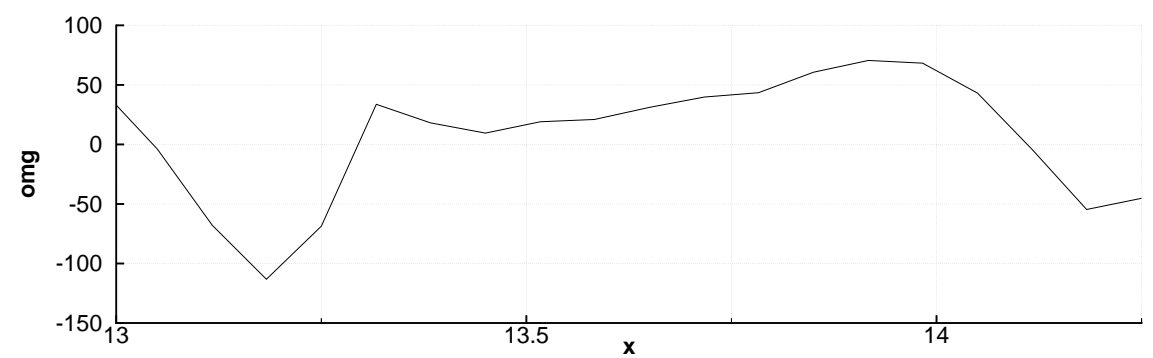

c)

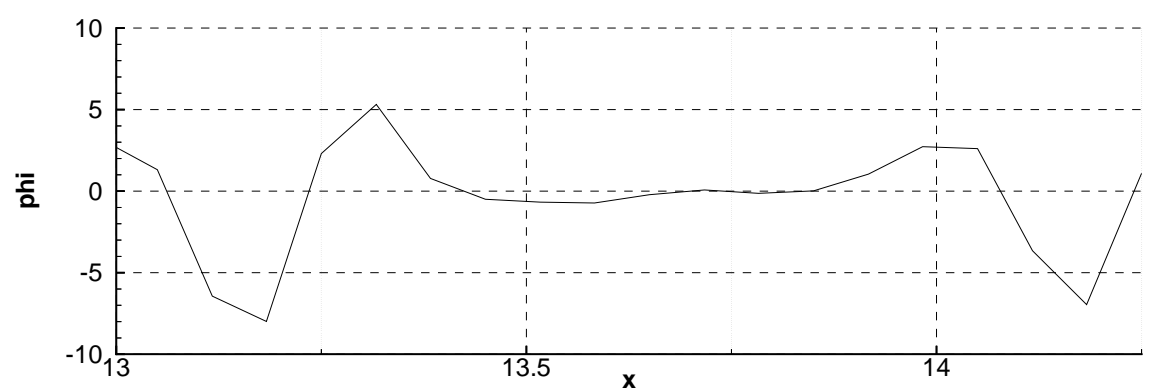

d)

Figure 4: a) : Stream-function; b): $C p$; c): wall vorticity; d) : vorticity flux 

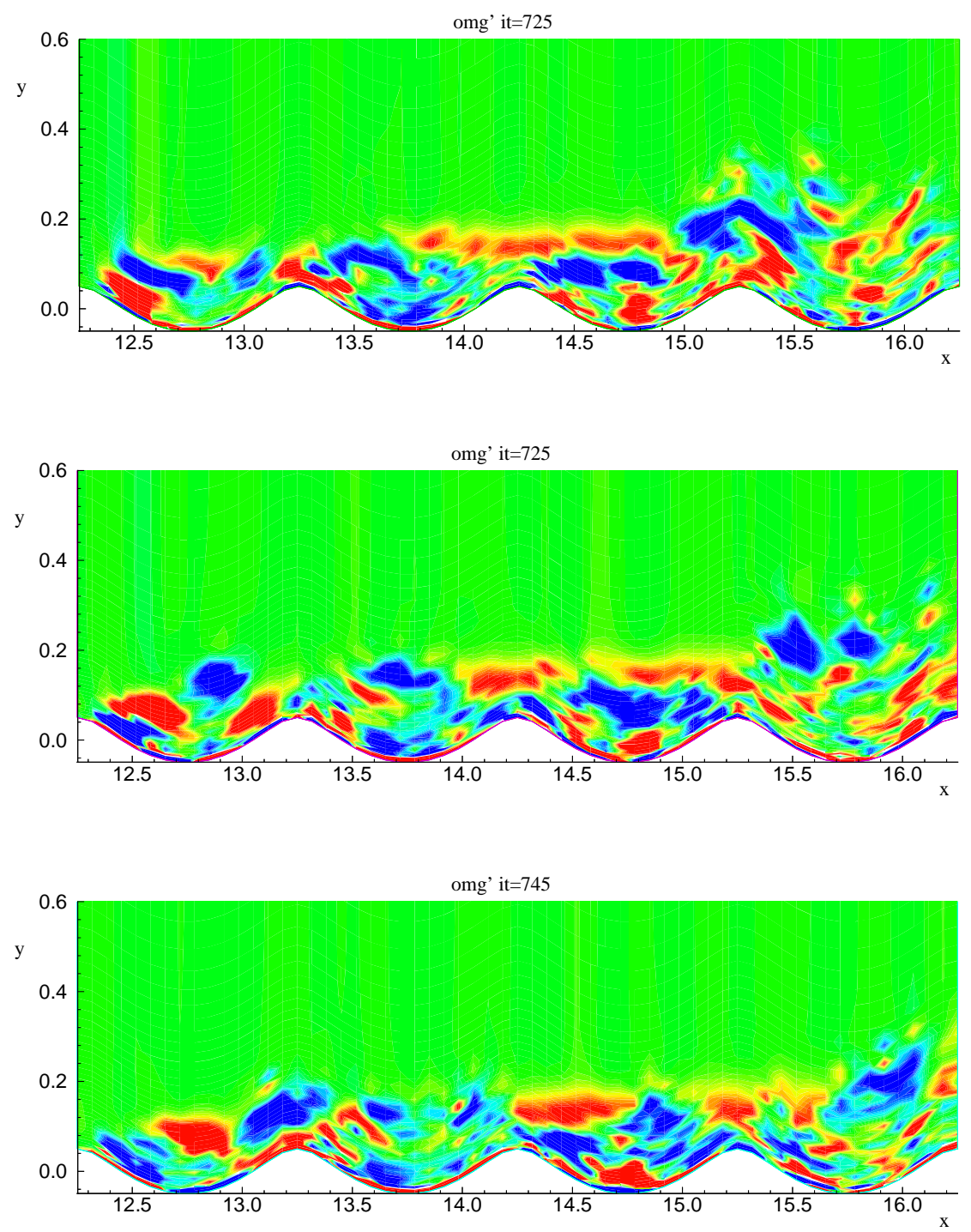

Figure 5: Vorticity disturbance, $\tau=32.25 \mathrm{~s}, \tau=36.75 \mathrm{~s}, \tau=37,25 \mathrm{~s}$. Blue is positive vorticity, and red is negative vorticity 